University of Nebraska - Lincoln

DigitalCommons@University of Nebraska - Lincoln

Faculty Publications from the Department of Electrical \& Computer Engineering, Department Electrical and Computer Engineering

2011

\title{
Fast growth of graphene patterns by laser direct writing
}

\author{
J. B. Park \\ University of Nebraska-Lincoln \\ W. Xiong \\ University of Nebraska-Lincoln \\ Y. Gao \\ University of Nebraska-Lincoln \\ M. Qian \\ University of Nebraska-Lincoln \\ Z. Q. Xie \\ University of Nebraska-Lincoln
}

See next page for additional authors

Follow this and additional works at: https://digitalcommons.unl.edu/electricalengineeringfacpub

Part of the Computer Engineering Commons, and the Electrical and Computer Engineering Commons

Park, J. B.; Xiong, W.; Gao, Y.; Qian, M.; Xie, Z. Q.; Mitchell, M.; Zhou, Y. S.; Han, G. H.; Jiang, L.; and Lu, Yongfeng, "Fast growth of graphene patterns by laser direct writing" (2011). Faculty Publications from the Department of Electrical and Computer Engineering. 225.

https://digitalcommons.unl.edu/electricalengineeringfacpub/225

This Article is brought to you for free and open access by the Electrical \& Computer Engineering, Department of at DigitalCommons@University of Nebraska - Lincoln. It has been accepted for inclusion in Faculty Publications from the Department of Electrical and Computer Engineering by an authorized administrator of DigitalCommons@University of Nebraska - Lincoln. 


\section{Authors}

J. B. Park, W. Xiong, Y. Gao, M. Qian, Z. Q. Xie, M. Mitchell, Y. S. Zhou, G. H. Han, L. Jiang, and Yongfeng Lu 


\title{
Fast growth of graphene patterns by laser direct writing
}

\author{
J. B. Park, ${ }^{1}$ W. Xiong, ${ }^{1}$ Y. Gao, ${ }^{1}$ M. Qian, ${ }^{1}$ Z. Q. Xie, ${ }^{1}$ M. Mitchell, ${ }^{1}$ Y. S. Zhou, ${ }^{1}$ G. H. Han, ${ }^{2}$ \\ L. Jiang, ${ }^{3}$ and Y. F. Lu ${ }^{1, a)}$ \\ ${ }^{1}$ Department of Electrical Engineering, University of Nebraska-Lincoln, Lincoln, Nebraska 68588-0511, USA \\ ${ }^{2}$ Department of Physics, Sungkyunkwan University, Suwon, 440-746, South Korea \\ ${ }^{3}$ Department of Mechanical and Automation Engineering, Beijing Institute of Technology, Beijing 100081, \\ People's Republic of China
}

(Received 26 December 2010; accepted 3 March 2011; published online 22 March 2011)

\begin{abstract}
Rapid single-step fabrication of graphene patterns was developed using laser-induced chemical vapor deposition (LCVD). A laser beam irradiates a thin nickel foil in a $\mathrm{CH}_{4}$ and $\mathrm{H}_{2}$ environment to induce a local temperature rise, thereby allowing the direct writing of graphene patterns in precisely controlled positions at room temperature. Line patterns can be achieved with a single scan without pre- or postprocesses. Surprisingly, the growth rate is several thousand times faster than that of general CVD methods. The discovery and development of the LCVD growth process provide a route for the rapid fabrication of graphene patterns for various applications. (C) 2011 American Institute of Physics. [doi:10.1063/1.3569720]
\end{abstract}

Graphene is a fast-growing topic in science and technology in the past few years. Even more attention has been attracted after the discoverers of graphene won the 2010 Nobel Prize in Physics. But what makes graphene such an exciting topic for researchers? It is because graphene is a really promising material in emerging electronic technology due to the ease of manipulation (2-dimensional sheet can be used with current planar manufacturing processes) and their unique electrical, ${ }^{1}$ mechanical, ${ }^{2,3}$ thermal, ${ }^{4}$ and optical properties. ${ }^{5}$ Graphene is expected to be the core material for flexible and transparent electronics, ${ }^{6}$ including displays, solar cells, sensors, and electrodes. Up to now, graphene can be obtained through several different approaches such as mechanical exfoliation, ${ }^{7,8}$ annealing of $\mathrm{SiC},{ }^{9,10}$ and chemical vapor deposition (CVD). ${ }^{11-14}$

Among the methods for graphene fabrication, CVD is the most promising method due to the benefits of large-scale production of high quality graphene and patterning process for various electronic applications. ${ }^{11}$ Especially, large-scale patterns can be achieved by patterning of catalytic metal films before growth ${ }^{12}$ or direct patterning of graphene after growth. ${ }^{13}$ Although the CVD method can achieve large production volume using a roll-to-roll method, ${ }^{14}$ the fabrication of graphene patterns by CVD methods is a multistep process which is time consuming and costly. Generally, CVD methods consist of several steps including annealing, growth, cooling, and patterning process for fabrication graphene patterns, and the growth process needs several minutes up to a few hours. ${ }^{11-14}$ Besides, the patterning process also requires expensive masks and time consuming processes. Therefore, more efforts are needed to make the conventional CVD techniques more suitable for electronic applications.

In this work, we developed a rapid single-step approach for fabrication of graphene patterns by laser-induced CVD (LCVD). Line patterns were fabricated on precisely controlled positions on a nickel foil. Localized laser heating on the nickel foil resulted in a rapid local temperature rise at the laser focal point; and the temperature dropped rapidly after

\footnotetext{
a) Author to whom correspondence should be addressed. Electronic mail: ylu2@unl.edu. Tel.: (402) 472-8323. FAX: (402) 472-4732.
}

the laser beam moved away, which is much faster than that in conventional thermal CVD methods. The growth rate is three orders of magnitude faster than that of the conventional thermal CVD methods. Graphene patterns can be fabricated through the single-step process without annealing and patterning procedures. Finally, the graphene patterns on the nickel foil could be transferred onto a $\mathrm{SiO}_{2} / \mathrm{Si}$ substrate for optical characterization and potential device applications.

The growth of graphene patterns was conducted in a stainless steel vacuum chamber. As shown in Fig. 1, a focused continuous wave laser beam (optically pumped solid state laser; Coherent Co. G5, $\lambda=532 \mathrm{~nm}$ ) was used as a heat source to increase the local temperature on the nickel foil in the stainless steel chamber. The chamber was initially evacuated to a base pressure of $10^{-3}$ torr with a rotary pump. $\mathrm{CH}_{4}$ was used as the precursor gas with a supply of $\mathrm{H}_{2}$; and the pressure of the chamber was kept at 500 torr during experiments. After many trials, 10 SCCM (SCCM denotes cubic centimeter per minute at STP) $\mathrm{CH}_{4}$ and $5 \mathrm{SCCM} \mathrm{H_{2 }}$ were selected as the optimal flow rates during experiments. The laser beam was then normally directed to the nickel foil with

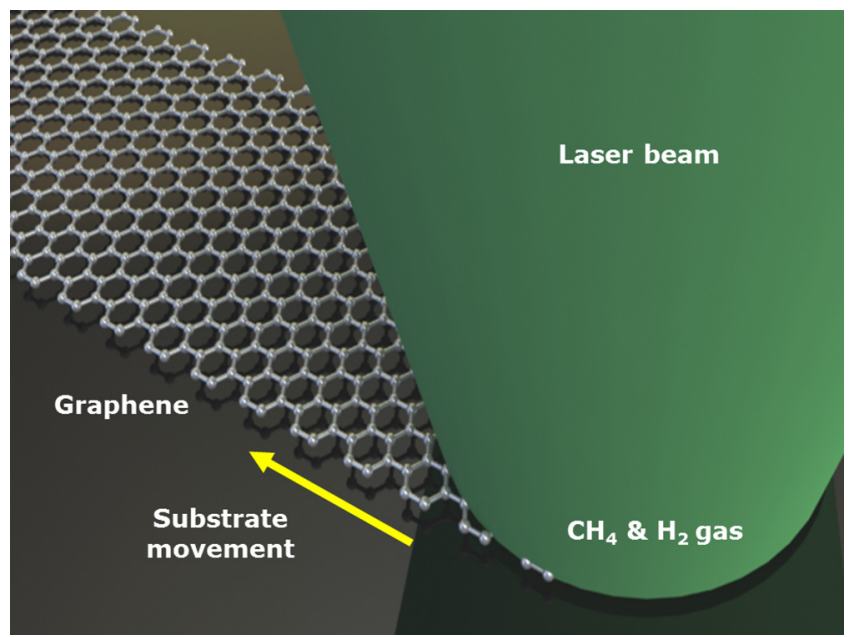

FIG. 1. (Color online) Illustration of direct writing of graphene patterns by LCVD on a nickel foil. 

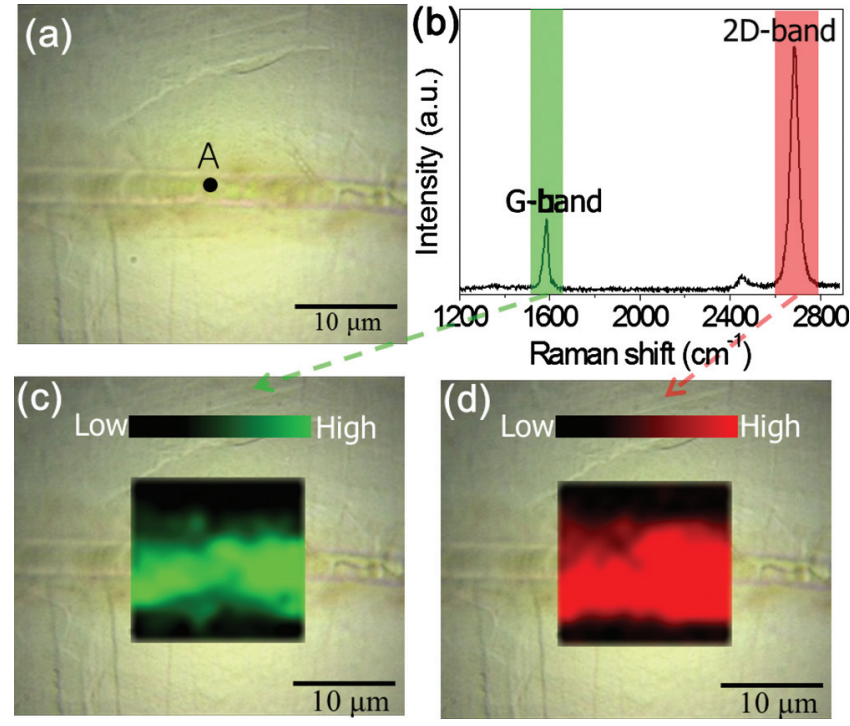

FIG. 2. (Color) (a) Optical micrograph of a graphene pattern on a nickel foil and (b) its Raman spectrum at "A" region. Raman intensity mapping of (c) G-bnad $\left(1582 \mathrm{~cm}^{-1}\right)$ and (d) 2D-band $\left(2691 \mathrm{~cm}^{-1}\right)$ (wavelength $=514.5 \mathrm{~nm})$.

an objective lens (Mitutoyo Corp., NIR 5×, focal length $=40 \mathrm{~mm}$ ). The laser beam was defocused on the nickel foil with a spot diameter of $20 \mu \mathrm{m}$ to reduce the power sensitivity. Laser power was controlled at about $5 \mathrm{~W}$ to induce the local temperature rise for graphene growth. For direct writing of graphene patterns, the substrate was moved precisely by a motorized stage with respect to the laser beam. The scan speed of the motorized stage was around $50 \mu \mathrm{m} / \mathrm{s}$.

For transferring graphene to $\mathrm{SiO}_{2} / \mathrm{Si}$ substrates, graphene-patterned nickel foils were first spin-coated with a poly(methyl methacrylate) (PMMA) layer at $2500 \mathrm{rpm}$. The nickel foils in nickel/graphene/PMMA layers were then wet etched using a commercially available etchant (nickel etchant Type 1, Transience Co.) for 30-60 min, resulting in a graphene/PMMA film floating on the etchant. This film was then collected manually and rinsed in de-ionized water before transferring it onto a $\mathrm{SiO}_{2} / \mathrm{Si}$ substrate (with a $300 \mathrm{~nm}$ $\mathrm{SiO}_{2}$ top layer). Finally, the PMMA layer in the graphene/ PMMA layers was dissolved in acetone, leaving the graphene patterns on the $\mathrm{SiO}_{2} / \mathrm{Si}$ substrates.

Figure 2(a) shows the optical micrograph of a line pattern of graphene which was fabricated by LCVD on a nickel foil. It is not easy to distinguish between the graphene pattern and the Ni substrate because the graphene pattern is highly transparent. Figure 2(b), Raman spectrum of region "A" in Fig. 2(a), clearly shows the evidence of a monolayer graphene. Both the symmetric 2D-band $\left(2691 \mathrm{~cm}^{-1}\right)$ with an full width at half maximum of $\sim 35 \mathrm{~cm}^{-1}$ and the high ratio of the peak intensities of 2D- to G-bands $\left(1582 \mathrm{~cm}^{-1}\right)$ $($ ratio $=3-4)$ confirm the presence of a monolayer graphene pattern. ${ }^{11-14}$ The absence of D-band in this spectrum also confirms the high quality of the as-grown graphene. Figures 2(c) and 2(d) show the intensity mapping of G-band (green) and 2D-band (red) as shown in Fig. 2(b), respectively. The Raman mapping in Figs. 2(c) and 2(d) shows that the intensities of the G- and 2D-band signals are clearly distinguished from the surrounding nonirradiated areas, thus demonstrating the precisely controlled locality of the graphene pattern in the LCVD process. The Raman intensity distributions of the graphene patterns are relatively uniform in the laserirradiated areas, also demonstrating the uniformity of the fabricated graphene patterns.

It is noteworthy that the graphene patterns were fabricated with a single scan on the nickel foil without annealing, cooling, or other supportive procedures such as mask patterning. A $10 \mu \mathrm{m}$ wide line pattern was directly written at a scan speed of $\sim 50 \mu \mathrm{m} / \mathrm{s}$ on the nickel foil. This means that the graphene pattern with a size of $10 \times 10 \mu \mathrm{m}^{2}$ can be rapidly grown in $0.2 \mathrm{~s}$, which implies that the growth rate is several thousand times faster than that of conventional thermal CVD methods (up to $20 \mathrm{~min}$ ). ${ }^{11,13}$ It is believed that the rapid heating and cooling rates at the local region of the metal foil contribute to the rapid growth of graphene patterns. The fast heating and cooling processes play significant roles in the rapid growth of graphene patterns and can enhance the graphene growth. ${ }^{12,15}$ The heating and cooling processes for a $10 \times 10 \mu \mathrm{m}^{2}$ area (a unit area of graphene pattern) have to be finished within $0.2 \mathrm{~s}$ because the line patterns with $10 \mu \mathrm{m}$ wide are fabricated at the scan speed of $50 \mu \mathrm{m} / \mathrm{s}$ (the cooling rate faster than $5000{ }^{\circ} \mathrm{C} / \mathrm{s}$ ). These heating and cooling processes for a unit area of graphene pattern are also several thousand times shorter than those of conventional thermal CVD methods. Considering inelastic electron-phonon coupling [( $\sim$ ps (Ref. 16)], a focused laser beam with high intensity can increase the local temperature of the nickel foil very rapidly up to the growth temperature $\left(1000{ }^{\circ} \mathrm{C}\right.$ in CVD methods, $\left.{ }^{11-14}\right)$. After laser irradiation, the local temperature can also drop rapidly through thermal conduction in the entire nickel foil placed in a room temperature environment. Therefore, localized laser heating has much faster heating and cooling rates than those in conventional thermal CVD methods using global heating and cooling process. The single-step fabrication of graphene patterns by LCVD utilizes a rapid heating and cooling mechanism in highly localized regions, which can dramatically reduce the process time. The graphene patterns were only observed following the laser scanning path on the illuminated-side of nickel foil, which is different from conventional CVD methods, in which graphene layers are observed on both sides of the foil due to the global heating.

Figures 3(a) and 3(b) show the optical micrographs of the graphene layers on a $\mathrm{SiO}_{2} / \mathrm{Si}$ substrate. The inset in Fig. 3(a) shows the line patterns which consist of both monolayer and multilayer graphene. The thin top $\mathrm{SiO}_{2}$ layer under the graphene patterns can enhance the optical sensitivity to human eyes, which allows characterizing the number of graphene layers. ${ }^{17}$ Color difference can be observed with respect to the number of graphene layers on the $\mathrm{SiO}_{2}$ layer as shown in Figs. 3(a) and 3(b). The color of the monolayer graphene pattern in Fig. 3(a) is lighter than that of the multilayer one in Fig. 3(b). The monolayer graphene pattern in Fig. 3(a) is highly transparent and its Raman spectrum in Fig. 3(c) also confirms a monolayer of the as-transferred graphene. Figure 3(d) shows the Raman spectra of the "b" and "c" regions in Fig. 3(b), which represents the bilayer and multilayer characteristics of the graphene, respectively. It should also be noted that the experimental condition to produce monolayer graphene patterns is very sensitive to the scan speed. A lower scan speed is more suitable for the fabrication of multilayer graphene.

Atomic force microscopy (AFM) was carried out to study the surface topography of the graphene patterns on the 

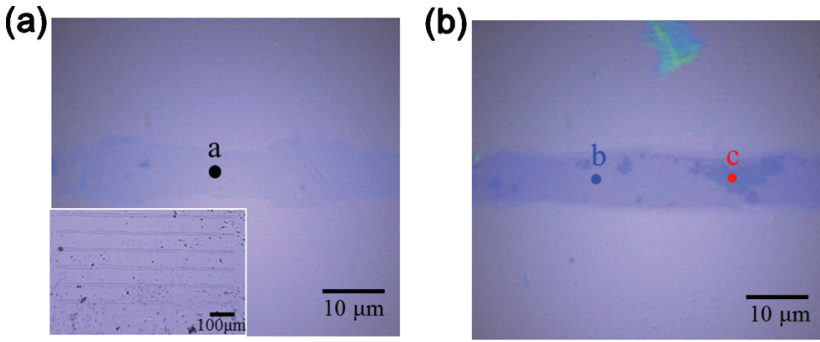

(c)

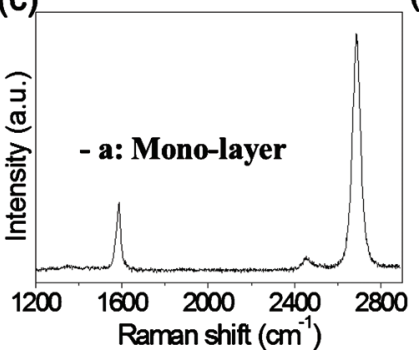

(d)

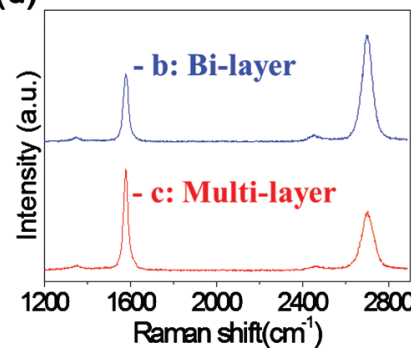

FIG. 3. (Color) Optical micrographs of (a) monolayer graphene and (b) multilayer graphene pattern on $\mathrm{SiO}_{2} / \mathrm{Si}$ wafer $\left(\mathrm{SiO}_{2}=300 \mathrm{~nm}\right)$. The inset in Fig. 3(a) shows the line patterns which consist of both monolayer and multilayer graphene. (c) Raman spectrum of the monolayer graphene at "a" region. Raman spectra of bilayer and multilayer graphene patterns at "b" and "c" region, respectively. (Excitation wavelength $=514.5 \mathrm{~nm}$ ).

$\mathrm{SiO}_{2}$ substrates. The graphene patterns have a crumpled topography and can be easily torn off, as shown in Fig. 4. The crumpled graphene is caused by the difference thermal expansion between graphene patterns and the nickel substrate during cooling process. ${ }^{18}$ The width difference along the patterns is attributed to a different temperature distribution along the width direction, which results from nonuniform reflectivity of the nickel foil during the laser beam scanning. The nanoparticles in Fig. 4 are PMMA residues, which are not completely removed during the transfer processing. Figure 4(b), the magnified scan image of the selected region of "A" in Fig. 4(a), shows the edge area of the line pattern. It indicates that the outer region of the graphene pattern is higher than the inner region, which indicates that the edge of the graphene is lifted up from the substrate. The bottom inset shows the surface profile across points "a" and "b." The
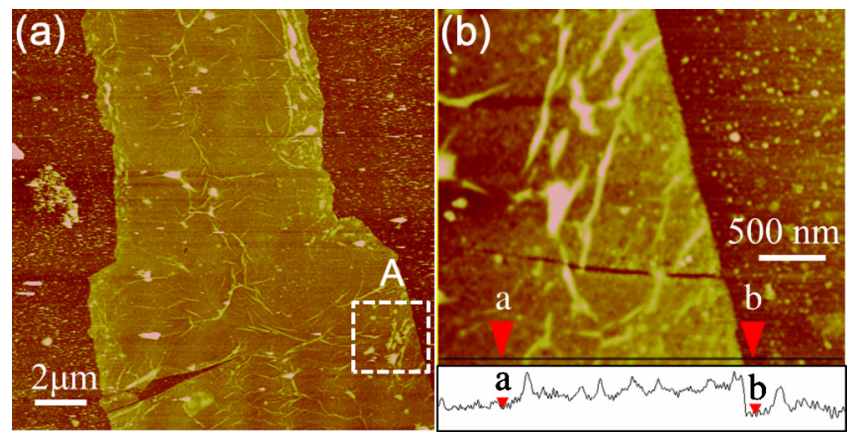

FIG. 4. (Color online) Surface topography of a graphene pattern on a $\mathrm{SiO}_{2} / \mathrm{Si}$ wafer. (a) AFM image of the graphene pattern. (b) The magnified image of a selected region in "A." The bottom inset shows the surface profile across (a) and (b). Note that the height difference between "a" and "b" is $0.4 \mathrm{~nm}$. height difference between "a" and "b" is $0.4 \mathrm{~nm}$, which corresponds to a monolayer graphene thickness, but most of regions of graphene patterns are usually crumpled and lifted up from the substrate, which makes it difficult to figure out the exact thickness of the graphene patterns. ${ }^{19}$

In conclusion, a rapid single-step fabrication method was developed for graphene patterning using LCVD. Line patterns of graphene were grown on nickel foils at room temperature with a short process time. No annealing or pattering processes are required for the fabrication of graphene patterns on nickel foils. The significantly enhanced growth rate of graphene is ascribed to the rapid heating and cooling mechanism in the LCVD process. Graphene pattern of 10 $\times 10 \mu \mathrm{m}^{2}$ area can be rapidly grown in $0.2 \mathrm{~s}$, which implies that the graphene growth rate is three orders of magnitude faster than the conventional thermal CVD methods. The fabricated line patterns can be transferred to various substrates for different applications, without additional steps removing the graphene from the back-side of nickel foil. It is believed that the LCVD method for graphene patterns will have a significant contribution to the rapid-developing graphene industry.

This research work was financially supported by National Science Foundation (Grant Nos. ECCS 0652905 and CMMI 0758199) and Nebraska Research Initiative.

${ }^{1}$ A. K. Geim and K. S. Novoselov, Nature Mater. 6, 183 (2007).

${ }^{2}$ C. Lee, X. Wei, J. W. Kysar, and J. Hone, Science 321, 385 (2008).

${ }^{3}$ S. Bunch, S. S. Verbridge, J. S. Alden, A. M. Zande, J. M. Parpia, H. G. Craighead, and P. L. McEuen, Nano Lett. 8, 2458 (2008).

${ }^{4}$ R. Prasher, Science 328, 185 (2010).

${ }^{5}$ F. Bonaccorso, Z. Sun, T. Hasan, and A. C. Ferrari, Nat. Photonics 4, 611 (2010).

${ }^{6}$ J. K. Wassei and R. B. Kaner, Mater. Today 13, 52 (2010).

${ }^{7}$ K. S. Novoselov, D. Jiang, F. Schedin, T. J. Booth, V. V. Khotkevich, S. V. Morozov, and A. K. Geim, Proc. Natl. Acad. Sci. U.S.A. 102, 10451 (2005).

${ }^{8}$ P. Blake, P. D. Brimicombe, R. R. Nair, T. J. Booth, D. Jiang, F. Schedin, L. A. Ponomarenko, S. V. Morozov, H. F. Gleeson, E. W. Hill, A. K. Geim, and K. S. Novoselov, Nano Lett. 8, 1704 (2008).

${ }^{9}$ P. Sutter, Nature Mater. 8, 171 (2009).

${ }^{10}$ V. Y. Aristov, G. Urbanik, K. Kummer, D. V. Vyalikh, O. V. Molodtsova, A. B. Preobrajenski, A. A. Zakharov, C. Hess, T. Hänke, B. Büchner, I. Vobornik, J. Fujii, G. Panaccione, Y. A. Ossipyan, and M. Knupfer, Nano Lett. 10, 992 (2010).

${ }^{11}$ X. Li, W. Cai, J. An, S. Kim, J. Nah, D. Yang, R. Piner, A. Velamakanni, I. Jung, E. Tutuc, S. K. Banerjee, L. Colombo, and R. R. Ruoff, Science 324, 1312 (2009).

${ }^{12}$ K. S. Kim, Y. Zhao, H. Jang, S. Y. Lee, J. M. Kim, K. S. Kim, J.-H. Ahn, P. Kim, J.-Y. Choi, and B. H. Hong, Nature (London) 457, 706 (2009).

${ }^{13}$ Y. Lee, S. Bae, H. Jang, S. Jang, S.-E. Zhu, S. H. Sim, Y. I. Song, B. H. Hong, and J.-H. Ahn, Nano Lett. 10, 490 (2010).

${ }^{14}$ S. Bae, H. Kim, Y. Lee, X. Xu, J.-S. Park, Y. Zheng, J. Balakrishnan, T. Lei, H. R. Kim, Y. I. Song, Y.-J. Kim, K. S. Kim, B. Özyilmaz, J.-H. Ahn, B. H. Hong, and S. Iijima, Nat. Nanotechnol. 5, 574 (2010).

${ }^{15}$ J. B. Park, S. H. Jong, M. S. Jeong, S. C. Lim, and Y. H. Lee, Nanotechnology 20, 185604 (2009).

${ }^{16}$ M. A. Al-Nimr, Int. J. Thermophys. 18, 1257 (1997).

${ }^{17}$ P. Blake, E. W. Hill, A. H. Castro Neto, K. S. Novoselov, D. Jiang, R. Yang, T. J. Booth, and A. K. Geim, Appl. Phys. Lett. 91, 063124 (2007).

${ }^{18}$ X. Li, Y. Zhu, W. Cai, M. Borsiak, B. Han, D. Chen, R. D. Piner, L. Colombo, and R. S. Ruoff, Nano Lett. 9, 4359 (2009).

${ }^{19}$ C. Soldano, A. Mahmood, and E. Dujardin, Carbon 48, 2127 (2010). 